\title{
Theoretical and experimental lifetime and oscillator strength determination in singly ionized neodymium (Nd II)
}

\author{
H. L. Xu, ${ }^{1}$ S. Svanberg, ${ }^{1}$ R. D. Cowan, ${ }^{2}$ P.-H. Lefèbvre, ${ }^{3}$ P. Quinet ${ }^{3,4}$ \\ and E. Biémont ${ }^{3,4 \star}$ \\ ${ }^{1}$ Department of Physics, Lund Institute of Technology, PO Box 118, S-221 00 Lund, Sweden \\ ${ }^{2}$ Los Alamos National Laboratory, Los Alamos, USA \\ ${ }^{3}$ IPNAS, Université de Liège, Sart Tilman B15, B-4000 Liège, Belgium \\ ${ }^{4}$ Astrophysique et Spectroscopie, Université de Mons-Hainaut, B-7000 Mons, Belgium
}

Accepted 2003 August 6. Received 2003 July 14; in original form 2003 May 14

\begin{abstract}
Radiative lifetime measurements were performed with time-resolved laser-induced fluorescence techniques for 24 levels of $\mathrm{Nd}$ II in the energy range $20500-32500 \mathrm{~cm}^{-1}$. For 17 levels, no previous experimental data exist. These results have allowed the testing of new theoretical calculations with the relativistic Hartree-Fock method taking configuration interactions and core-polarization effects into account, and a satisfying agreement has been found for this complex ion. A new set of calculated oscillator strengths, accurate within a few per cent for the strongest transitions, is presented for 107 lines of astrophysical interest appearing in the wavelength range $358.0-1100.0 \mathrm{~nm}$. These results will be useful to evaluate abundance values of neodymium in chemically peculiar stars in relation with cosmochronology.
\end{abstract}

Key words: atomic data - atomic processes - stars: chemically peculiar.

\section{INTRODUCTION}

The consideration of the r-process abundance patterns in some stars allows an age determination of the earliest galactic halo. In particular, the comparison of the abundances of the heavy elements (i.e. thorium and uranium) synthesized soon after the formation of our Galaxy with those of lighter stable neutron capture elements allows the estimation of the age of this halo. However, some singly ionized rare-earths, interesting for cosmochronometry (in relation with the third n-capture peak), have only poorly determined transition probabilities (Sneden, Lawler \& Cowan 2002). As a consequence, narrowing the uncertainties in age estimates relies clearly on further improvements in r-process theory but also on high-resolution spectroscopy of very metal-poor stars itself depending upon accurate atomic data.

The age of some very metal-poor stars (see e.g. Cayrel et al. 2001) can be estimated, by comparison of abundance ratios of radioactive and stable nuclear species, using different pairs of elements such as $\mathrm{U} / \mathrm{Th}, \mathrm{U} / \mathrm{Os}$ and U/Ir. Nd II is one of the elements which can be used as a stable nuclear species in cosmochronology for estimating the age of the Galaxy (Lawler, Whaling \& Grevesse 1990). The present study is mainly aimed at obtaining a new set of atomic data for this ion having in mind contribution to the cosmochronometry based on the pair $\mathrm{U} / \mathrm{Nd}$.

^E-mail: E.Biemont@ulg.ac.be
In a more general context, accurate atomic data for lanthanide ions are required for refining the stellar elemental abundances and for more firmly establishing the odd-even effect observed in many stars: in fact the elements with an even atomic number appear generally more abundant than the odd elements. In some stars, however, this odd-even effect does not appear clearly because some of the lines (which are frequently only a few of them) used for deducing abundance values are affected by blending with the abundant elements (iron-group elements) and by possible unknown hyperfine structure effects.

Singly ionized neodymium is frequently observed in astrophysical spectra and the analysis of $\mathrm{Nd}$ II lines leads to strong overabundance estimates of this element in many stars compared to the adopted solar system value. Nd II lines have been identified in Ap stars, e.g. by Smith \& Lambert (1985), in Bp stars, e.g. by Cowley $\&$ Crosswhite (1978) and some Nd II lines appear very strong in the stars of the Cr-Eu-Sr subgroup (Adelman 1973) and in Am stars (Smith 1973, 1974). Nd II lines have also been identified in G- and K-type dwarfs (Gilroy et al. 1988), in Ba stars (Lambert 1985) and in S-type stars (Bidelman 1953). Recently, the presence of $\mathrm{Nd}$ in HgMn stars has been investigated by Dolk et al. (2002) who found a strong overabundance of this element. $\mathrm{Nd}$ II is also observed in the solar photospheric spectrum (see e.g. Gorshkov \& Komarovskii 1986; Anders \& Grevesse 1989).

A number of investigations have been performed to improve our knowledge of the radiative parameters of $\mathrm{Nd}$ II and to obtain oscillator strength values of astrophysical interest. Corliss \& 
Bozman (1962) first contributed to the f value determination using arc spectra; however, these values have been proven to be very uncertain and even wrong sometimes by an order of magnitude. This point has been frequently discussed in the literature. Maier \& Whaling (1977) reported branching fraction (BF) measurements for the known transitions originating from nine levels of $\mathrm{Nd}$ II by investigating a hollow-cathode discharge in argon, and oscillator strengths were deduced from a combination of the new BFs and previous lifetime measurements carried out by Andersen et al. (1975). Using these data, an abundance value of neodymium in the Sun was derived from the analysis of 12 weak Nd II solar lines (Maier \& Whaling 1977). However, as pointed out by Simonsen et al. (1988), the assumption of LTE is questioned for arc or hollow-cathode discharges and is responsible of large errors affecting some of the previous measurements.

In order to refine the solar content of Nd, Ward et al. (1984, 1985) measured the lifetimes of eight and 24 levels in Nd II, respectively, using the laser-ion beam technique, and deduced oscillator strengths of some Nd II lines from a combination of the BFs deduced from arc spectra (Corliss \& Bozman 1962; Reader et al. 1980) or measured by Maier \& Whaling (1977) and of the new lifetime values.

The lifetime compilation of Blagoev \& Komarovskii (1994) contains 11 lifetime values originating from the delayed-coincidence measurements of Gorshkov et al. (1982). Additional measurements of radiative lifetimes in $\mathrm{Nd}$ II have been carried out by Pinciuc et al. (2001) who considered 35 levels up to $30000 \mathrm{~cm}^{-1}$ using the collinear beam-laser method and by Lu et al. (1989) and Shi et al. (1991), who concentrated on the odd level at $23537 \mathrm{~cm}^{-1}(J=$ 9/2) using the time-resolved collinear fast-beam laser approach. The most recent results in the same ion were undertaken by Scholl et al. (2002), who measured 13 lifetime values using two variants of the beam-laser method.

Although one way of obtaining accurate $f$ values results from a combination of experimental BFs and experimental lifetimes, it is extremely difficult in the lanthanide elements due to their very complex atomic structures and spectra and to the problem associated with eventual missing branches (e.g. for infrared transitions). Instead, an alternative way of deducing absolute oscillator strengths results from the combination of accurate experimental lifetimes with theoretical BFs which are in turn very difficult to calculate accurately. As they could be affected by large uncertainties, a detailed check by comparison with experiment appears necessary. This procedure has been applied with success for many lanthanide ions as illustrated on the web site at the address http://www.umh.ac.be/ astro/dream.shtml (Quinet et al. 2002; Biémont \& Quinet 2003) and has been adopted in the present study.

In the present paper, we report on lifetime measurements of 24 levels of $\mathrm{Nd}$ II, of which 17 are considered for the first time, using time-resolved laser-induced fluorescence spectroscopy on a laserproduced plasma. Also, theoretical calculations of lifetimes, using a relativistic Hartree-Fock approach (HFR method; Cowan 1981) taking core-polarization effects and configuration interactions into account, are reported in the present paper. New oscillator strengths are proposed for 107 transitions originating from the levels from which lifetimes have been measured in the present work. The aim of this contribution is to provide new radiative parameters for transitions emitted from low energy levels (the highest energy considered being $32465 \mathrm{~cm}^{-1}$ ) which are the most susceptible to be observed in astrophysical spectra and to make reliable abundance determinations, based on a more extended set of lines possible (data on 94 new lines were obtained).

\section{THE SPECTRUM OF ND II}

Nd II has seven stable isotopes: ${ }^{142} \mathrm{Nd}(27.2$ per cent $),{ }^{143} \mathrm{Nd}(12.2$ per cent), ${ }^{144} \mathrm{Nd}(23.8$ per cent $),{ }^{145} \mathrm{Nd}$ (8.3 per cent),${ }^{146} \mathrm{Nd}(17.2)$, ${ }^{148} \mathrm{Nd}(5.7$ per cent $)$ and ${ }^{150} \mathrm{Nd}(5.6$ per cent $)$. The ground-state level of this ion is $4 f^{4}\left({ }^{5} \mathrm{I}\right) 6 \mathrm{~s}{ }^{6} \mathrm{I}_{7 / 2}$. The nuclear spin of the isotopes 143 and 145 is $7 / 2$, these two odd-mass stable isotopes being affected by hyperfine structure (Lu Fuquan et al. 1991; Ma Hongliang et al. 1997; Li Maosheng et al. 2000).

The energy levels of Nd II, as compiled by the NIST (Martin, Zalubas \& Hagan 1978, http://physics.nist.gov/cgi-bin/AtData/ main_asd), were obtained from previous investigations carried out by Albertson, Harrison \& McNally (1942) and Schuurmans (1946) and from more recent analyses of Nd I and Nd II spectra performed at the Zeeman Laboratory of Amsterdam and at the Laboratoire Aimé Cotton in Paris (Wyart 1968; Hoekstra 1969; Blaise et al. 1970; Morillon 1970; Blaise et al. 1971). A revised interpretation of the $\mathrm{Nd}$ II spectrum, with help of the ${ }^{144} \mathrm{Nd}-{ }^{150} \mathrm{Nd}$ isotope shift, has been made by Blaise \& Wyart (1984) who considered the complete system of levels of $\mathrm{Nd}$ II built on the core $\mathrm{f}^{3}$, proposed 126 new levels and revised 96 previous values. This new analysis relied upon parametric studies of the low-lying subconfigurations. The energy levels used in the present work (see Section 4) are basically those taken from the NIST compilation (Martin et al. 1978; http://physics.nist.gov/cgi-bin/AtData/ main_asd) and from the analysis of Blaise \& Wyart (1984). The experimentally determined even levels belong to the configurations $4 f^{4} 6 s, 4 f^{4} 5 d, 4 f^{3} 5 d 6 p$ and $4 f^{3} 6 s 6 p$, while the odd levels belong to the configurations $4 f^{3} 5 d^{2}, 4 f^{3} 5 d 6 s$ and $4 f^{4} 6 p$. It should be emphasized, however, that the designation of many levels is still uncertain and that most of them show strong configuration interaction mixings.

\section{MEASUREMENTS OF RADIATIVE LIFETIMES}

Radiative lifetimes of 24 low-energy levels of $\mathrm{Nd}$ II belonging to the $4 f^{3} 5 d^{2}$ and $4 f^{4} 6 p$ configurations were measured using the method of time-resolved laser-induced fluorescence. The $\mathrm{Nd}^{+}$ions produced by laser ablation, using a pulsed Nd:YAG laser focused on to a rotating neodymium target, were excited by single-step excitation from the ground state and different metastable states. The levels investigated, whose energy values are taken from the NIST atomic spectra data base, are presented in Table 1 (see also Tables 2 and 3).

In order to generate the required excitation pulses, a seeder injected Nd:YAG laser (Continuum NY-82), characterized by a 532$\mathrm{nm}$ wavelength, an 8 -ns pulse duration and a $400-\mathrm{mJ}$ pulse energy, was used to pump a dye laser (Continuum Nd-60). However, before entering the dye laser, the beam from the Nd:YAG laser was sent into a compressor, based on stimulated Brillouin scattering in water, to shorten the laser pulses to about $1 \mathrm{~ns}$. The dye laser, operated with a 4-dicyanomethylene-2-methyl-6p-dimethylaminostyryl-4H-pyran (DCM) dye, was effectively frequency doubled in a potassium dihydrogen phosphate (KDP) crystal. According to the excitation requirements shown in Table 1, different orders of Stokes (S) and anti-Stokes (A) Raman scattering from the fundamental and second harmonic beam from the dye laser were obtained by focusing the beam into a cell with hydrogen at at pressure of about 10 bar. The excitation light was then isolated with a $\mathrm{CaF}_{2}$ Pellin-Broca prism and sent into the vacuum chamber crossing a neodymium plasma at a distance of about $10 \mathrm{~mm}$ above the foil. The plasma was produced by focusing 532-nm wavelength 
Table 1. Nd II levels considered in the present work and excitation schemes. The excitation origin (level energy, in $\mathrm{cm}^{-1}$ ), the corresponding wavelength $\left(\lambda_{1}\right.$, in $\left.\mathrm{nm}\right)$ and the detection wavelength $\left(\lambda_{2}\right.$, in $\left.\mathrm{nm}\right)$, respectively, are given in the table. $2 \omega$ means the second harmonic, S and AS are written for the first Stokes and anti-Stokes components of the Raman scattering.

\begin{tabular}{|c|c|c|c|c|}
\hline \multicolumn{5}{|c|}{ Excitation Observed laser } \\
\hline $\mathrm{E}\left(\mathrm{cm}^{-1}\right)^{a}$ & Origin & $\lambda_{1}(\mathrm{~nm})_{\mathrm{vac}}$ & $\lambda_{2}(\mathrm{~nm})_{\mathrm{vac}}$ & Mode \\
\hline 20672.581 & 513.322 & 496.05 & 526 & $\omega+\mathrm{AS}$ \\
\hline 20830.048 & 1650.199 & 521.38 & 492 & $2 \omega+3 S$ \\
\hline 20907.332 & 1470.097 & 514.48 & 519 & $\omega+\mathrm{AS}$ \\
\hline 22696.885 & 513.322 & 450.78 & 509 & $2 \omega+2 S$ \\
\hline 23229.991 & 0.0 & 430.48 & 440 & $2 \omega+2 S$ \\
\hline 24721.059 & 1470.097 & 430.09 & 452 & $2 \omega+2 S$ \\
\hline 25044.675 & 1650.199 & 427.45 & 399 & $2 \omega+2 S$ \\
\hline 26640.085 & 0.0 & 375.37 & 383 & $2 \omega+\mathrm{S}$ \\
\hline 27245.453 & 513.322 & 374.08 & 391 & $2 \omega+\mathrm{S}$ \\
\hline 27425.015 & $\begin{array}{c}0.0 \\
513.322\end{array}$ & $\begin{array}{l}364.63 \\
371.59\end{array}$ & 385 & $\begin{array}{l}2 \omega+S \\
2 \omega+S\end{array}$ \\
\hline 27445.854 & 3801.917 & 422.94 & 402 & $2 \omega+2 S$ \\
\hline 27744.196 & 1470.097 & 380.60 & 397 & $2 \omega+S$ \\
\hline 27921.395 & 513.322 & 364.86 & 381 & $2 \omega+S$ \\
\hline 28213.885 & $\begin{array}{r}513.322 \\
1650.199\end{array}$ & $\begin{array}{l}361.00 \\
376.45\end{array}$ & 376 & $\begin{array}{l}2 \omega+\mathrm{S} \\
2 \omega+\mathrm{S}\end{array}$ \\
\hline 28285.619 & 1470.097 & 372.92 & 389 & $2 \omega+S$ \\
\hline 28563.485 & 1470.097 & 369.09 & 392 & $2 \omega+S$ \\
\hline 29043.459 & 1650.199 & 365.05 & 385 & $2 \omega+S$ \\
\hline 29434.270 & 2585.453 & 372.46 & 390 & $2 \omega+S$ \\
\hline 29484.600 & 1650.199 & 359.27 & 379 & $2 \omega+S$ \\
\hline & 3066.75 & 378.53 & & $2 \omega+S$ \\
\hline 30094.850 & 2585.453 & 363.51 & 391 & $2 \omega+S$ \\
\hline & 3801.917 & 380.33 & & $2 \omega+\mathrm{S}$ \\
\hline 30246.771 & 3801.917 & 378.15 & 397 & $2 \omega+\mathrm{S}$ \\
\hline 30707.282 & 3801.917 & 371.67 & 390 & $2 \omega+S$ \\
\hline 31153.870 & 3801.917 & 365.60 & 375 & $2 \omega+S$ \\
\hline 32464.640 & 5085.619 & 365.24 & 378 & $2 \omega+S$ \\
\hline
\end{tabular}

laser pulses, emitted from a 10-ns duration Nd:YAG laser (Continuum Surelite) with variable pulse energy in the range $2-10 \mathrm{~mJ}$, on a rotating neodymium foil, which is placed in a vacuum chamber with $10^{-6}-10^{-5}$ mbar background pressure. Both Nd:YAG lasers, operated in an external trigger mode, were controlled by a digital delay generator (Stanford Research Systems Model 535).

Fluorescence from the levels investigated was collected by a fused silica lens, and then appropriately filtered by a 1/8 m monochromator (resolution of $6.4 \mathrm{~nm} \mathrm{~mm}{ }^{-1}$ ). The strongest signal from the upper levels to possible lower levels was selected and detected by a Hamamatsu R3809U-58 photomultiplier tube (200 ps rise time). The time-resolved signal from the detector was captured by means of a digital oscilloscope (Tektronix Model DSA 602), where an average of 1000 pulses was performed to obtain a sufficiently high, smooth signal-to-noise ratio. A Thorlabs SV2-FC photodiode (120 ps rise time), driven by the excitation beam, was employed to trigger the oscilloscope. Through a GPIB cable, the time-resolved fluorescence decay data were transferred to a personal computer and the lifetime evaluation was performed by an exponential fit. A sample of a decay curve is shown in Fig. 1. For more details about the method, see e.g. Biémont et al. (2001) and Zhang et al. (2002).

In the measurements, possible systematic errors were considered. The plasma density and temperature in the interaction region can be adjusted by changing the delay time between the excitation and ablation laser pulses. With appropriately varied plasma conditions, the

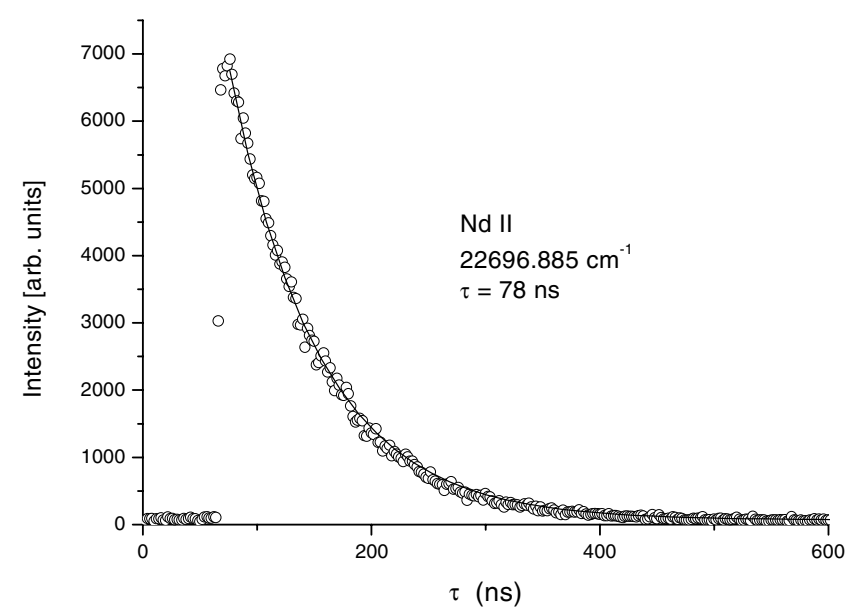

Figure 1. A typical $\mathrm{Nd}$ II experimental decay curve with an exponential fit. The lifetime for this level situated at $22696.885 \mathrm{~cm}^{-1}$ is 78(7) ns.

intensity of the fluorescence signal was changed by a factor of 10 , while the lifetimes remained constant within the experimental scattering. This confirmed that the collisional quenching and radiation trapping effects within the parameter range used were negligible. A magnetic field of about $100 \mathrm{G}$ provided by a pair of Helmholtz coils was added or removed over the plasma zone to check potential quantum-beat effects on long lifetimes. The entrance slit of the monochromator was opened in a maximum way in order to eliminate flight-out-of-view effects for longer lifetime measurements. About 10 fluorescence decay curves for each studied level were recorded under different experimental conditions. All experimental lifetime results are included in Table 2 with estimated statistical errors. They are also compared in the same table with previous experimental results and with the theoretical lifetimes as calculated in the present work (see Section 4).

The new lifetime values agree within the estimated uncertainties with the recent measurements of Pinciuc et al. (2001) (four measurements in common) obtained using the collinear beam-laser method. No comparison was possible with the recent results of Scholl et al. (2002) because there are no levels in common between the two papers. A good agreement is also observed with the laser measurements of Ward et al. (1985). For the level at $27445.854 \mathrm{~cm}^{-1}$, however, a discrepancy of a factor of 2 is observed. As our measurement for this level entirely supports the value reported by Pinciuc et al. (2001), it is reasonable to believe that the problem is originating from the result published by Ward et al. (1985). Some discrepancies do appear when comparing the laser measurements (this work and Ward et al. 1985) with the delayed coincidence results of Gorshkov et al. (1982), this technique appearing sometimes unreliable in singly ionized atoms (for a recent discussion, see e.g. Li et al. 1999).

\section{HFR CALCULATIONS}

The theoretical method considered in this paper for obtaining the required BFs is the well established HFR approach described by Cowan (1981). Configuration interaction was explicitly retained among the $4 f^{3} 5 d^{2}, 4 f^{3} 5 d 6 s, 4 f^{3} 6 s^{2}$ and $4 f^{4} 6 p$ odd-parity configurations and among the $4 f^{4} 6 s, 4 f^{4} 5 d, 4 f^{3} 5 d 6 p$ and $4 f^{3} 6 s 6 p$ even-parity configurations. Due to the numerous possible interactions between the low-lying configurations that could not be considered explicitly in the calculations in view of the computer limitations, corepolarization effects were introduced in the model by considering 
Table 2. Nd II observed and calculated lifetime values and comparison with previous results.

\begin{tabular}{lccc}
\hline & \multicolumn{3}{c}{ Lifetime (ns) } \\
$\mathrm{E}\left(\mathrm{cm}^{-1}\right)^{g}$ & \multicolumn{2}{c}{ This work } & Previous \\
& Exp. & Theory $^{f}$ & \\
\hline 20672.581 & $79(7)$ & 65.7 & \\
20830.048 & $61(6)$ & & $80.6(4.7)^{a}$ \\
20907.332 & $109(14)$ & 100.3 & $14(3)^{b}, 19.5(0.9)^{c}$ \\
22696.885 & $78(7)$ & 93.2 & \\
23229.991 & $13.4(0.5)$ & 8.4 & $13.3(0.4)^{a}, 13(3)^{b}, 14.0(0.8)^{c}, 13(3)^{d}$ \\
24721.059 & $112(12)$ & & \\
25044.675 & $20.0(2.0)$ & 15.3 & $10(1)^{b}, 20.8(0.7)^{c}$ \\
26640.085 & $22.5(1.4)$ & 21.0 & \\
27245.453 & $23.7(1.7)$ & & \\
27425.015 & $29(2)$ & 21.2 & \\
27445.854 & $36(2)$ & & \\
27744.196 & $22.2(1.5)$ & 15.7 & $13.1(1.4)^{a}$ \\
27921.395 & $16.7(0.8)$ & 13.9 & \\
28213.885 & $31(2)$ & & $19(2)^{b}$ \\
28285.619 & $18.1(0.9)$ & 18.6 & \\
28563.485 & $18.1(1.4)$ & 21.2 & \\
29043.459 & $26.5(1.5)$ & & \\
29434.270 & $14.8(1.1)$ & 23.2 & \\
29484.600 & $14(1.2)$ & & \\
30094.850 & $40(2)$ & & \\
30246.771 & $12.7(0.7)$ & 7.5 & \\
30707.282 & $17.6(1.0)$ & 16.9 & \\
31153.870 & $31(2)$ & 16.1 & \\
32464.640 & $20.5(1.5)$ & & \\
\hline
\end{tabular}

Notes. ${ }^{a}$ Pinciuc et al. (2001), ${ }^{b}$ Gorshkov et al. (1982), ${ }^{c}$ Ward et al. (1985), ${ }^{d}$ Andersen et al. (1975). ${ }^{f}$ This work: HFR calculations with core-polarization included (see text). ${ }^{g}$ From NIST compilation (Martin et al. 1978 and http://physics.nist.gov/cgi-bin/AtData/main_asd). a core-polarization potential and a correction to the dipole operators according to a procedure described in previous papers (see e.g. Quinet et al. 1999). The polarizability of the ionic core was chosen to be equal to the value tabulated by Fraga, Karwowski \& Saxena (1976) for Nd IV, i.e. $\alpha_{\mathrm{d}}=8.30 \mathrm{au}$, and the cut-off radius was equal to $1.683 \mathrm{au}$, which corresponds to the HFR value of $\langle r\rangle$ for the outermost Xe-like core-orbitals $\left(5 \mathrm{p}^{6}\right)$.

In view of the unknown label of many experimentally determined energy levels available in $\mathrm{Nd}$ II and of the strong mixing occuring among many of the low-lying levels, no semi-empirical adjustment of the radial parameters could be seriously considered. Instead, in order to obtain a more realistic representation of the Nd II energy spectrum, all the Slater integrals were scaled down by a factor 0.75 , a factor recommended by Cowan (1981), and the average energies were adjusted to reproduce adequately the lowest levels identified in the NIST compilation (Martin et al. 1978; http://physics.nist.gov/cgibin/AtData/main_asd).

Looking at the theoretical results, it is worth mentioning that most of the levels considered in the present work are characterized by very strong mixings as illustrated in Table 3 . These strong perturbations can lead to difficulties for establishing unambiguously the correspondence between experimental and theoretical level values. This is the case for the levels situated at $20830.048,24721.059$, 27 245.453, 27 445.854, 28 213.885, 29 043.459, 29 484.600, 30 094.850 and $32464.640 \mathrm{~cm}^{-1}$, for which it was impossible to quote, with certainty, the corresponding HFR values even when comparing the available Landé factors. Consequently, no HFR lifetime values are reported in Table 2 for these levels.

The calculated lifetimes are compared with the experimental ones in Table 2. As seen from this table, an agreement within 30 per cent has been found between the HFR lifetimes and the laser measurements obtained in the present work for 9 levels. For the remaining

Table 3. Odd-parity energy levels in Nd II for which radiative lifetimes have been measured in the present work.

\begin{tabular}{|c|c|c|c|c|c|c|c|}
\hline $\begin{array}{l}\mathrm{E}_{\exp }^{a} \\
\left(\mathrm{~cm}^{-1}\right)\end{array}$ & $\mathrm{gexp}^{a}$ & Config. $^{a}$ & Term $^{a}$ & $J$ & $\begin{array}{l}\mathrm{E}_{\mathrm{HFR}}^{b} \\
\left(\mathrm{~cm}^{-1}\right)\end{array}$ & $\mathrm{g}_{\mathrm{HFR}}{ }^{b}$ & Leading components ${ }^{b, c}$ \\
\hline 20672.581 & 0.780 & & ${ }^{4} \mathrm{I}$ & $9 / 2$ & 21338 & 0.788 & $29 \% 4 f^{3} 5 d^{2}{ }^{4} I+16 \% 4 f^{3} 6 s^{2}{ }^{4} I+10 \% 4 f^{4} 6 p^{4} I$ \\
\hline 20907.332 & 0.890 & & ${ }^{4} \mathrm{~K}$ & $11 / 2$ & 20918 & 0.961 & $25 \% 4 f^{3} 5 d^{2}{ }^{4} K+12 \% 4 f^{3} 5 d^{26} G+7 \% 4 f^{3} 5 d^{2}{ }^{4} K$ \\
\hline 22696.885 & 0.965 & & ${ }^{4} \mathrm{I}$ & $11 / 2$ & 23212 & 1.005 & $28 \% 4 f^{3} 6 s^{2}{ }^{4} I+12 \% 4 f^{3} 5 d^{24} I+10 \% 4 f^{3} 5 d^{2}{ }^{4} I$ \\
\hline 23229.991 & 0.780 & $4 f^{4}\left({ }^{5} I\right) 6 p$ & ${ }^{6} \mathrm{~K}$ & $9 / 2$ & 23522 & 0.699 & $51 \% 4 f^{4} 6 p^{6} K+6 \% 4 f^{3} 5 d^{24} I+3 \% 4 f^{3} 5 d^{2}{ }^{4} I$ \\
\hline 24721.059 & 1.050 & & ${ }^{4} \mathrm{I}$ & $13 / 2$ & & & \\
\hline 27245.453 & 0.955 & $4 f^{4}\left({ }^{5} I\right) 6 p$ & ${ }^{4} \mathrm{~K}$ & $11 / 2$ & & & \\
\hline 27425.015 & 1.170 & $4 f^{4}\left({ }^{5} \mathrm{I}\right) 6 \mathrm{p}$ & ${ }^{6} \mathrm{H}$ & $9 / 2$ & 27568 & 1.098 & $16 \% 4 f^{4} 6 p^{6} H+11 \% 4 f^{3} 5 d 6 s^{6} H+7 \% 4 f^{3} 5 d^{2}{ }^{4} G$ \\
\hline 27445.854 & 1.110 & & & $15 / 2$ & & & \\
\hline 27744.196 & 1.080 & $4 f^{4}\left({ }^{5} I\right) 6 p$ & ${ }^{6} \mathrm{I}$ & $13 / 2$ & 27864 & 1.149 & $25 \% 4 f^{4} 6 p^{6} I+22 \% 4 f^{3} 5 d^{2}{ }^{6} H+4 \% 4 f^{3} 5 d^{2}{ }^{4} K$ \\
\hline 27921.395 & 1.035 & $4 f^{4}\left({ }^{5} \mathrm{I}\right) 6 \mathrm{p}$ & ${ }^{4} \mathrm{I}$ & $9 / 2$ & 27660 & 0.898 & $28 \% 4 f^{4} 6 p^{4} I+7 \% 4 f^{3} 5 d 6 s^{4} I+4 \% 4 f^{3} 5 d^{2}{ }^{6} D$ \\
\hline 28213.885 & 0.720 & $4 f^{4}\left({ }^{5} I\right) 6 p$ & ${ }^{4} \mathrm{H}$ & $7 / 2$ & & & \\
\hline 29484.600 & 1.022 & $4 f^{4}\left({ }^{5} \mathrm{I}\right) 6 \mathrm{p}$ & ${ }^{4} \mathrm{I}$ & $11 / 2$ & & & \\
\hline 30094.850 & 1.105 & $4 f^{4}\left({ }^{5} \mathrm{I}\right) 6 \mathrm{p}$ & ${ }^{4} \mathrm{~K}$ & $15 / 2$ & & & \\
\hline 30246.771 & 1.220 & $4 f^{4}\left({ }^{5} \mathrm{I}\right) 6 \mathrm{p}$ & ${ }^{6} \mathrm{I}$ & $17 / 2$ & 30446 & 1.252 & $54 \% 4 f^{4} 6 p^{6} I+6 \% 4 f^{3} 5 d^{26} I+5 \% 4 f^{3} 5 d^{24} K$ \\
\hline 30707.282 & 1.205 & $4 f^{4}\left({ }^{5} \mathrm{I}\right) 6 \mathrm{p}$ & ${ }^{6} \mathrm{H}$ & $15 / 2$ & 31108 & 1.178 & $22 \% 4 f^{4} 6 p^{6} H+12 \% 4 f^{3} 5 d 6 s^{6} H+12 \% 4 f^{3} 5 d 6 s^{4} L$ \\
\hline 31153.870 & 1.115 & $4 f^{4}\left({ }^{5} \mathrm{I}\right) 6 \mathrm{p}$ & ${ }^{4} \mathrm{I}$ & $13 / 2$ & 31042 & 1.091 & $24 \% 4 f^{4} 6 p^{4} I+8 \% 4 f^{3} 5 d 6 s^{4} I+7 \% 4 f^{3} 5 d 6 s^{4} K$ \\
\hline 32464.640 & 1.130 & $4 f^{4}\left({ }^{5} I\right) 6 p$ & ${ }^{4} \mathrm{I}$ & $15 / 2$ & & & \\
\hline
\end{tabular}

Notes. ${ }^{a}$ From NIST compilation (Martin et al. 1978 and http://physics.nist.gov/cgi-bin/AtData/main_asd). ${ }^{b}$ Present work. ${ }^{c}$ Only the main components are given. 
Table 4. Oscillator strengths $(\log g f)$ and transition probabilities $(\mathrm{gA})$ of the most intense lines $(\log g f>-1.50)$ depopulating the levels considered in the present work. $\lambda(\mathrm{nm})$ is the transition wavelength in $\mathrm{nm}$. $E_{\text {low }}$ and $E_{\text {up }}$ correspond to the lower and upper excitation energies involved in the transition. log $g f$ and $g A$ are the weighted oscillator strengths and transition probabilities. 6.807(7) is written for $6.80710^{7} \mathrm{~s}^{-1}$.

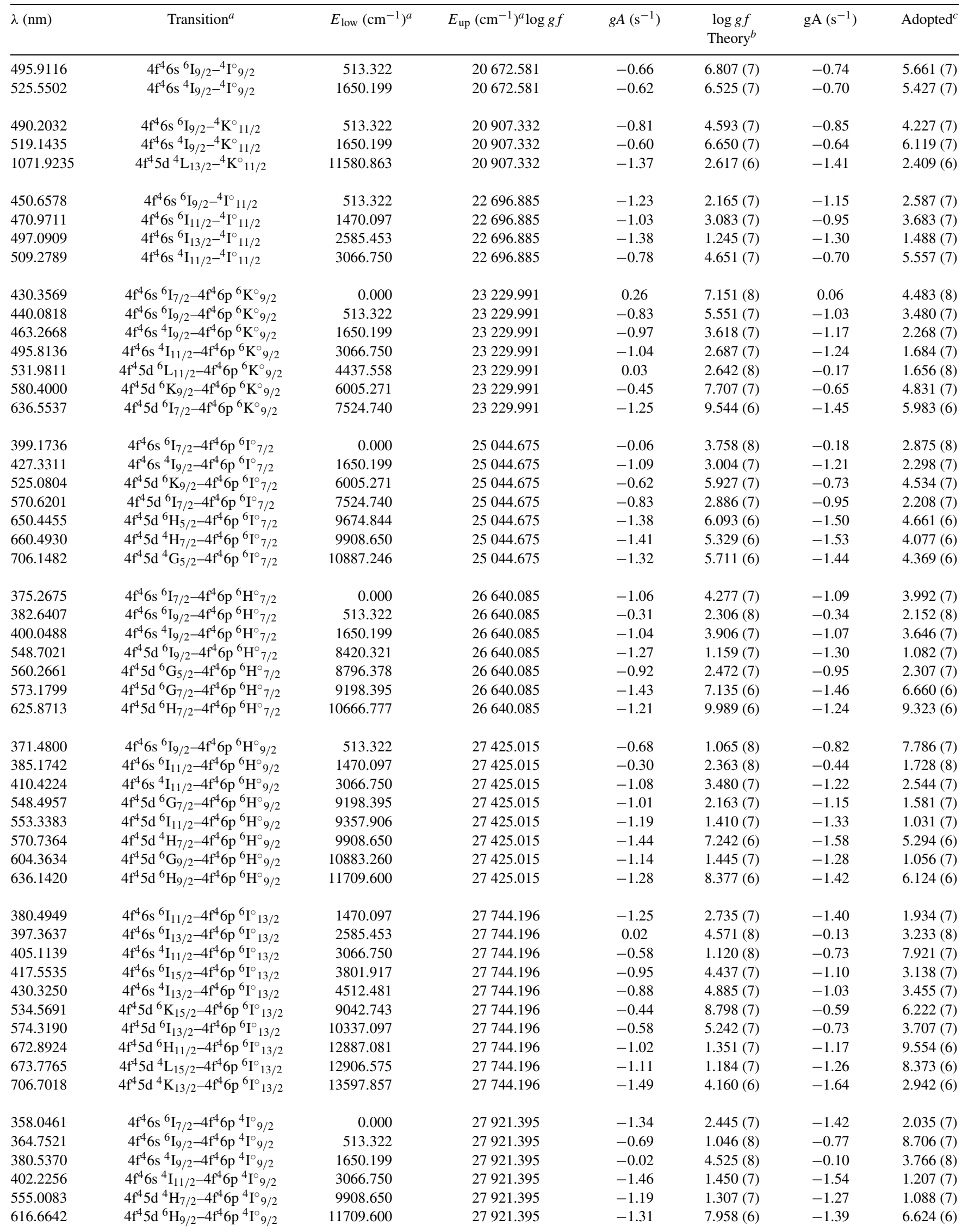


Table 4 - continued

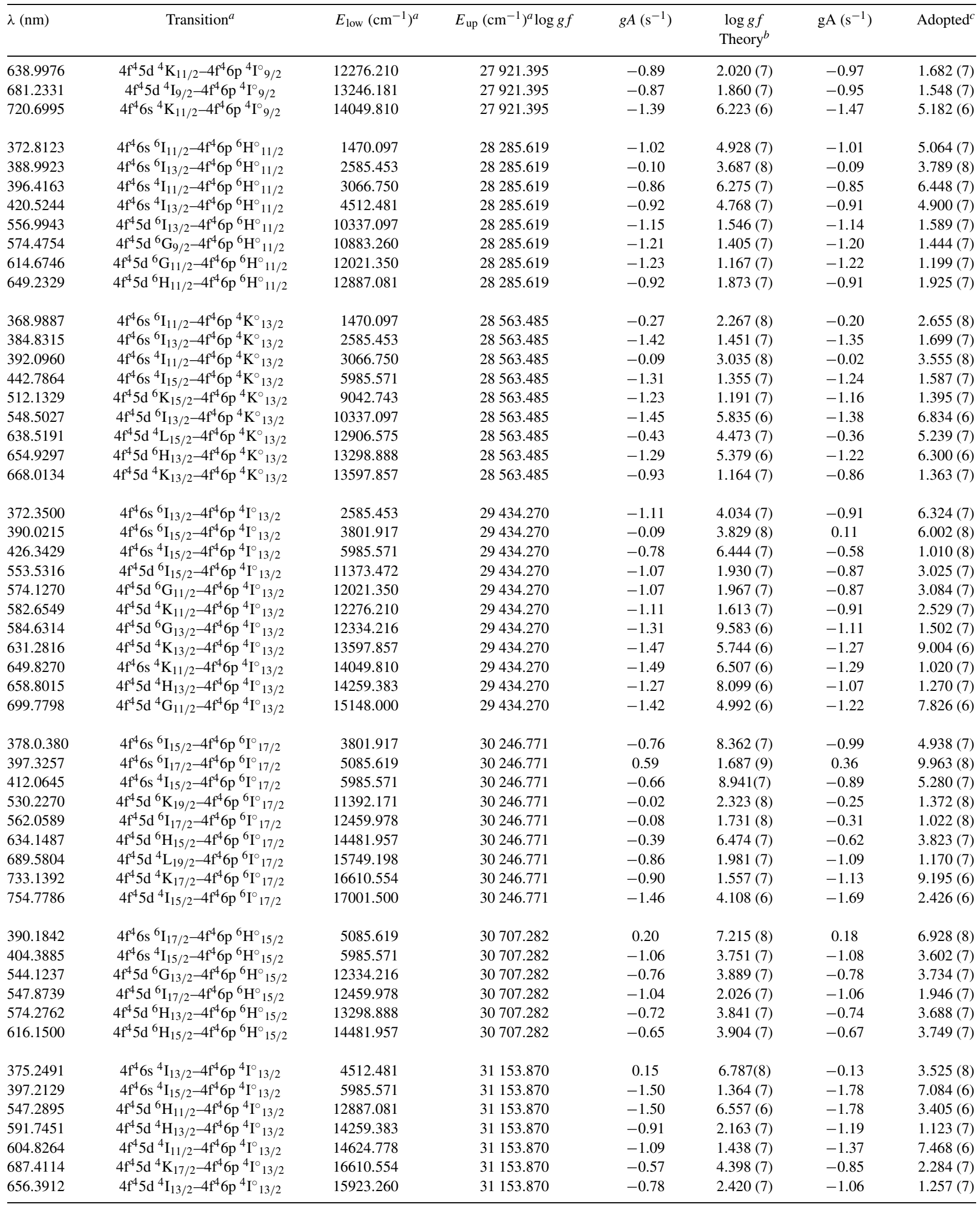

Notes. ${ }^{a}$ According to the NIST compilation. ${ }^{b}$ This work: HFR theoretical results (see the text). ${ }^{c}$ This work: results deduced from the experimental lifetime values and from the theoretical HFR branching fractions. 
6 levels at 23 229.991, 27 425.015, 27 744.196, 29 434.270, 30246.771 and $31153.870 \mathrm{~cm}^{-1}$, however, larger discrepancies between theory and experiment are observed. These disagreements are attributed to the strong mixing and to inaccurate eigenvalue compositions (see Table 3). More accurate results for these six levels would probably require larger expansion sets for the wavefunctions but further investigations in that direction were prevented basically by computer limitations.

We have extracted from our computer output the weighted oscillator strengths $(\log g f$, where $g$ is the statistical weight of the lower level) and transition probabilities ( $g A$, where $g$ is the statistical weight of the upper level) for the transitions depopulating the 15 levels for which calculated lifetimes are given in Table 2. These are reported in Table 4 for the strongest $(\log g f>-1.5)$ lines. Using the experimental lifetime values as measured in the present work and the theoretical BFs, as calculated with the HFR approach, it was possible to deduce 'corrected' $f$ values and transition probabilities for the 107 visible or ultraviolet $(358.0<\lambda<1100.0 \mathrm{~nm})$ transitions listed in Table 4 under the heading 'adopted'.

Transition probabilities available for Nd II are very sparse and a comparison of our theoretical data with experiment is only possible with the BFs obtained by Maier \& Whaling (1977) for the two multiplets common to the two works i.e. for the transitions originating from the levels at 23229.991 and $30246.771 \mathrm{~cm}^{-1}$. For the strongest transitions of the first multiplet, the agreement theoryexperiment is better than 10 per cent, larger discrepancies appearing for the weak line at $440.0828 \mathrm{~nm}$ and for the very weak lines at 463.2688 and $495.8139 \mathrm{~nm}$. For the second multiplet, the comparison has little meaning because only five transitions are quoted in the work of Maier \& Whaling (1977) while nine transitions with $\log g f>-1.50$ are given in our Table 4 . The missing transitions in the first work are probably responsible of the discrepancies observed between the two works, particularly for the transition at $378.0391 \mathrm{~nm}$.

In the present work, a new set of transition probabilities has been obtained for 107 transitions from a combination of laser lifetime measurements and branching fraction calculations. For 94 transitions, there were no oscillator strengths previously available. The new oscillator strengths are expected to be characterized by an uncertainty of a few $(\leqslant 10)$ per cent for the strongest transitions. As the transitions for which new $f$ values are proposed are originating from low excitation levels, the results presented in Table 4 are expected to help astrophysicists in their analysis of the high resolution astrophysical spectra now currently available and, consequently, in the determination of the chemical composition of CP stars. These results will be incorporated in the data base DREAM accessible at the web site: http://www.umh.ac.be/ astro/ dream.shtml.

In view of the complexity and of the richness of the $\mathrm{Nd}$ II spectrum, it is clear, however, from the present work that much additional effort is still needed for improving our knowledge of the radiative parameters (lifetimes, branching fractions and transition probabilities) in this spectrum of astrophysical interest.

\section{ACKNOWLEDGMENTS}

This work was financially supported by the Swedish Natural Science Research Council and by the EU-TMR access to Large-Scale Infrastructure Programme (contract HPRI-CT-1999-00041). Financial support from the Belgian FNRS is acknowledged by two of us (EB and PQ).

\section{REFERENCES}

Adelman S. J., 1973, ApJS, 26, 1

Albertson W. E., Harrison G. R., McNally J. R., Jr, 1942, Phys. Rev., 61, 167

Anders E., Grevesse N., 1989, Geoch. Cosmochim. Acta, 53, 197

Andersen T., Poulsen O., Ramanujam T. S., Petkov A. P., 1975, Sol. Phys., 44, 257

Bidelman W. P., 1953, ApJ, 117, 377

Biémont E., Quinet P., 2003, Phys. Scr. T, 105, 38

Biémont E., Palmeri P., Quinet P., Paquin G., Zhang Z. G., Somesfalean G., Svanberg S., 2001, MNRAS, 328, 1085

Blagoev K. B., Komarovskii V. A., 1994, Atomic Data Nucl. Data Tables, 56,1

Blaise J., Wyart J.-F., 1984, Phys. Scr., 29, 119

Blaise J., Chevillard J., Vergès J., Wyart J.-F., 1970, Spectrochim. Acta, Part $\mathrm{B}, 25,333$

Blaise J., Wyart J.-F., Hoekstra R., Kruiver P. J. G., 1971, J. Opt. Soc. Am., 61,1335

Cayrel R. et al., 2001, Nat, 409, 691

Corliss C. H., Bozman W. R., 1962, NBS Monograph No. 53

Cowan R. D., 1981, The Theory of Atomic Structure and Spectra. Univ. California Press, Berkeley CA

Cowley C. R., Crosswhite H. M., 1978, PASP, 90, 198

Dolk L., Wahlgren G. M., Lundberg H., Li Z. S., Litzén U., Ivarsson S., Ilyin I., Hubrig S., 2002, A\&A, 385, 111

Fraga S., Karwowski J., Saxena K. M. S., 1976, Handbook of Atomic Data. Elsevier, Amsterdam

Gilroy K. K., Sneden C., Pilachowski C., Cowan J. J., 1988, ApJ, 327, 298

Gorshkov V. N., Komarovskii V. A., 1986, SvA, 30, 333

Gorshkov V. N., Komarovskii V. A., Osherovich A. L., Penkin N. P., 1982, Astrophys. USSR, 17, 437

Hoekstra R., 1969, PhD thesis, Univ. Amsterdam

Lambert D. L., 1985, in Jaschek M., Keenan P. C., eds, Cool Stars with Excesses of Heavy Elements. Reidel, Dordrecht

Lawler J. E., Whaling W., Grevesse N., 1990, Nat, 346, 635

Maosheng L., Hongliang M., Miaohua C., Fuquam L., Jiayong T., Fujia Y., 2000, Phys. Rev. A, 62, 052504

Li Z. S., Svanberg S., Quinet P., Tordoir X., Biémont E., 1999, J. Phys. B, 32,1731

Lu F., Wu S., Shi W., Yang J., Song L., Tang J., Yang F., 1989, Chin. Phys. Lett., 6, 161

Fuquan L. et al., 1991, Phys. Rev. A, 44, 1843

Hongliang M., Wei S., Bin Y., Yong L., Dufei F., Fuquan L., Jiayong T., Fujia Y., 1997, J. Phys. B, 30, 3355

Maier R. S., Whaling W., 1977, J. Quant. Spectrosc. Radiat. Transfer, 18, 501

Martin W. C., Zalubas R., Hagan R., 1978, Atomic Energy Levels, The Rare Earth Elements. National Bureau of Standards, US DEprament of Commerce, Washington

Morillon C., 1970, Spectrochim. Acta, Part B, 25, 513

Pinciuc C. M., Rivest R. C., Izawa M. R., Holt R. A., Rosner S. D., Scholl T. J., 2001, Can. J. Phys., 79, 1159

Quinet P., Palmeri P., Biémont E., McCurdy M. M., Rieger G., Pinnington E. H., Wickliffe M. E., Lawler J. E., 1999, MNRAS, 307, 934

Quinet P., Palmeri P., Biémont E., Li Z. S., Zhang Z. G., Svanberg S., 2002, Journal of Alloys and Compounds, 344, 255

Reader J., Corliss C. H., Wiese W. L., Martin G. A., 1980, Wavelengths and Transition Probabilities for Atoms and Atomic Ions. National Bureau of Standards, US Department of Commerce, Washington

Scholl T. J., Holt R. A., Masterman D., Rivest R. C., Rosner S. D., Sharikova A., 2002, Can. J. Phys., 80, 713

Schuurmans Ph., 1946, Physica, 11, 419

Shi W., Lu F., Wu S., Shi P., Yang J., Song L., Tang J., Yang F., 1991, Phys. Rev. A, 43, 1451

Simonsen H., Worm T., Jessen P., Poulsen O., 1988, Phys. Scr., 38, 370

Smith M. A., 1973, ApJS, 25, 277

Smith M. A., 1974, ApJ, 189, 101 
Smith V. V., Lambert D. L., 1985, ApJ, 294, 326

Sneden C., Lawler J. E., Cowan J. J., 2002, Phys. Scr. T, 100, 15

Ward L., Vogel O., Ahnesjö A., Arnesen A., Hallin R., McIntyre L., Nordling C., 1984, Phys. Scr., 29, 551

Ward L., Vogel O., Arnesen A., Hallin R., Wännström A., 1985, Phys. Scr., 31,161
Wyart J.-F., 1968, PhD thesis, Univ. Paris

Zhang Z. G., Somesfalean G., Svanberg S., Palmeri P., Quinet P., Biémont E., 2002, A\&A, 384, 364

This paper has been typeset from a $\mathrm{TE}_{\mathrm{E}} \mathrm{L} / \mathrm{AT} \mathrm{E} \mathrm{X}$ file prepared by the author. 